\title{
Sulforaphane Treatment of Stress Urinary Incontinence Via the Nrf2-ARE Pathway in a Rat Model
}

\author{
Xiang Wan Chong Liu Yan-Bo Chen Meng Gu Zhi-Kang Cai Qi Chen \\ Zhong Wang \\ Department of Urology, Shanghai Ninth People's Hospital, Shanghai Jiao Tong University School of \\ Medicine
}

\section{Key Words}

Sulforaphane • Nrf2 • ARE • Stress Urinary Incontinence • Treatment

\begin{abstract}
Background/Aims: To explore the effect of sulforaphane (SFN) treatment in rats through the induction of Stress Urinary Incontinence (SUI) via the Nrf2-ARE pathway. Methods: A total of 18 female rats (Sprague-Dawley) were assigned to three groups: a control group, an SUI group, and an SUI+SFN group (six rats per group). Rats in the treatment groups were induced via postpartum vaginal balloon dilation and bilateral ovariectomy. Rats in the SUI+SFN group were treated via intraperitoneal injection once per day for a total of one month. Urethral sphincter muscle histological was observed by HE and Masson staining. Peak voiding pressure and interval of micturition were measured by cystometry. Oxidative stress markers and protein expression in the Nrf2-ARE pathway were examined by immunohistochemical staining and western blotting. Results: Prolonged micturition interval and higher peak voiding pressure were observed in the SUI+SFN group. Disturbance of muscle morphology was ameliorated, muscle content was elevated, and collagen content was restrained in response to SFN treatment. The SOD, GSH-Px, and CAT activities were elevated in the SUI+SFN group compared to those in the control group. The level of cell apoptosis was decreased in SUI rats after SFN treatment; however, apoptosis was mainly located in the urethral mucosa instead of the muscle layer. $\mathrm{SFN}$ reduced the $\mathrm{Bax} / \mathrm{Bcl}-2$ expression ratio. Nrf2 and Nrf2 target antioxidant proteins were elevated in the SFN group. Conclusions: SFN was effective for SUI treatment via decreasing oxidative stress and activating the Nrf2-ARE pathway.
\end{abstract}

X. Wan, C. Liu and Y.-B. Chen contributed equally to this work.

Qi Chen

and Zhong Wang

KARGER
Department of Urology, Shanghai Ninth People's Hospital,

Shanghai Jiaotong University School of Medicine, Shanghai 200011 (China)

E-Mail qiqi_chenqi@163.com, zhongwang2000@sina.com 


\section{Introduction}

Worldwide, more than 200 million people suffer from urinary incontinence (UI) [1]. The most common type of urinary incontinence is stress urinary incontinence (SUI), which has a disproportionally higher prevalence among women [2]. It has been reported that more than half of all women above 60 years of age suffer from SUI symptoms [3, 4].

Medication therapy for SUI has never been satisfactory [5]. Periurethral injection of bulking agents, such as collagen, cannot provide an ideal long-term outcome and may lead to complications, such as abscess formation, voiding dysfunction, and even pulmonary embolism [6, 7]. Various sling surgeries are more efficacious and prevalent but are associated with larvaceous complications, such as urinary tract injury and infection [8]. The exact regeneration mechanism of stem cells injection therapy is unknown so safety may be a problem, which is the limitation of stem cell therapy for SUI [9]..

Various explanations account for SUI. Vaginal delivery-caused birth trauma and menopause are widely recognized risk factors for SUI genesis in women [10]. A recent study implicated oxidative stress in the synergistic effect of birth trauma and menopauserelated SUI pathogenesis [11]. However, there are no reports of the treatment of SUI with sulforaphane (SFN), let alone studies explaining the exact mechanism. Since oxidative stress is a factor for SUI development [12-14], antioxidants may be effective for SUI treatment. Therefore, we intend to treat SUI in a novel manner.

Nrf2 is a key regulator of cellular responses to oxidative stress or electrophilic transcription via the promotion of antioxidative gene expression through the antioxidant response element (ARE) [15-17]. SFN is a nontoxic and phytochemical compound naturally obtained from cruciferous vegetables; particularly high concentrations of SFN have been derived from broccoli and broccoli sprouts $[18,19]$. The major mechanism of the antioxidative action of SFN is mediated by Nrf2, which combines with Kelch-like ECH-associated protein 1 (Keap1) within the cytoplasm under basal conditions [16]. SFN modifies the cysteines of Keap1, resulting in the translocation of Nrf2 to the nucleus, and subsequent binding to AREs leads to the expression of cytoprotective genes, such as heme oxygenase-1 (HO-1) and $\mathrm{NAD}(\mathrm{P}) \mathrm{H}$ : quinone oxidoreductase 1 (NQO1), to combat against electrophilic and oxidative stress $[20,21]$.

In the present study, we explored the role of SFN in treating SUI using an SUI rat model. In addition, we also investigated whether and how the Nrf2-ARE pathway is involved in SFN treatment of SUI.

\section{Materials and Methods}

\section{Reagents and Antibodies}

Sulforaphane was obtained from Cayman Chemical (USA). Anti-Nrf2, anti-HO-1, and anti-NQO1 antibodies were acquired from Abcam (Cambridge, UK); anti-Bcl-2, anti-Bax, anti-PCNA, and anti-GAPDH antibodies were obtained from CST (Danvers, MA, USA); and goat-anti-mouse IgG-HRP, goat-anti-rabbit IgG-HRP, and the DAB detection Kit were obtained from DAKO. The TdT-mediated dUTP Nick-End Labeling (TUNEL) Apoptosis Assay Kit was obtained from KeyGEN BioTECH (Nanjing, China). The BCA assay kit was obtained from Thermo Scientific. The nuclear protein extraction kit was obtained from the Beyotime Institute of Biotechnology (Beijing, China). The malondialdehyde (MDA) assay kit, the total superoxide dismutase (SOD) assay kit, the glutathione peroxidase (GSH-Px) assay kit, and the catalase (CAT) assay kit were obtained from the Nanjing Jiancheng Bioengineering Institute (Nanjing, China).

\section{SUI Model and Cystometry Preparation}

The Ethics Committee of the Shanghai Jiao Tong University School of Medicine approved all experimental instructions. Eighteen two-month-old primiparous female Sprague-Dawley rats, weighing 280 to $330 \mathrm{~g}$, at gestational day 16, were obtained from the Animal Center of the Ninth People's Hospital of the Shanghai Jiao Tong University School of Medicine. All SD rats were bred at two animals per cage with free access to tap water and food under conditions of $20-26^{\circ} \mathrm{C}$ and $40-60 \%$ relative humidity under a $12 \mathrm{~h}$ light/dark cycle. 


\section{Cellular Physiology Cell Physiol Biochem 2017;44:1912-1922

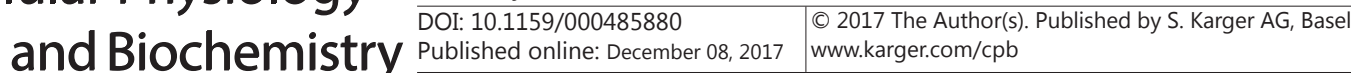 \\ Wan et al.: Treating Stress Urinary Incontinence}

We randomly divided the SD rats into three groups: a control group $(n=6)$, an SUI group $(n=6)$, and a SUI+SFN group $(n=6)$. The control group was subjected to control experiments. The SUI and SUI+SFN groups were subjected to the development of voiding dysfunction, using a previously published procedure $[10,22,23]$. Briefly, immediately after delivery, the rats were anesthetized via $10 \%$ chloral hydrate $(3 \mathrm{~mL} /$ $\mathrm{kg}$, intraperitoneally) and then fixed in a supine position. An intravaginal balloon filled with $4 \mathrm{~mL}$ of saline was introduced using a transurethral $12 \mathrm{~F}$ catheter, which was left in situ for $4 \mathrm{~h}$ to simulate prolonged labor. One week later, the rats were again anesthetized using chloral hydrate and underwent bilateral ovariectomy via bilateral back incision. Gentamycin $(1 \mathrm{~mL} / \mathrm{kg})$ was intramuscularly administered after surgery. From the first day after vaginal distention, we performed daily intraperitoneal injections of SFN $(0.5 \mathrm{mg} / \mathrm{kg})$ to rats in the SUI+SFN group. The control and SUI groups received the same volume of PBS with ethanol, since SFN was dissolved in ethanol and then diluted with PBS. The following experiments were conducted 30 days post vaginal distention: the rats in all groups were anesthetized via chloral hydrate $24 \mathrm{~h}$ prior to testing, and a catheter was placed in the bladder using a previously described procedure [24, 25]. A PE-50 polyethylene tube with a flared end, which served as an anchor, was inserted into the bladder dome. Subsequently, a 1-cm incision was made between the scapulae on the dorsum. The other end of the tube was pulled through the subcutaneous tunnel and out from the dorsum incision. We closed the dorsum incision using a purse string suture to fix the tube.

\section{Cystometric Analysis}

The cystometric analysis was performed as previously described by Liu et al. [26]. Continuous cystometry was performed in conscious rats in a metabolic cage without restraint. We connected the indwelling tube to a two-way valve. The valve was connected to an infusion pump and a pressure transducer. We ridded the system of any air bubbles to ensure continuous flow from the infusion pump. Saline was continuously infused into the bladder at a rate of $12 \mathrm{~mL} / \mathrm{h}$ at room temperature in all groups. We measured cystometric parameters, such as peak voiding pressure and interval of micturition. All rats were subsequently euthanized and the bladder-urethra complex was removed for further testing.

\section{Histological Examination and Immunohistochemical Staining}

Urethral sphincter specimens were fixed in 4\% paraformaldehyde, followed by paraffin embedding. The specimens were then processed and cut into sections with a thickness of $5 \mu \mathrm{m}$ for routine staining via hematoxylin and eosin (H\&E) and for IHC staining to observe the general morphology. Masson trichrome was performed to assess tissue fibrosis. We operated Immunohistochemical staining as previously reported. The $10 \mathrm{mM}$ sodium citrate buffer ( $\mathrm{pH} 6.0$ ) were added to the sections, and then were put to heat for antigen retrieval. As the instructions of the manufacturer, we applied primary antibodies, secondary antibodies, and DAB detection kit. Two pathologists evaluated histological tissue in a blinded manner.

\section{Masson's stain}

All samples were simultaneously stained by the same experimenter to prevent staining variations. Briefly, urethral tissue sections were fully immersed in Bouin's solution $\left(58^{\circ} \mathrm{C}\right)$ for $15 \mathrm{~min}$, followed by rinsing. We subsequently stained the urethral tissue sections with Weigert Hematoxylin for 10 min and rinsed the sections until only the nuclei remained stained. Biebrich Scarlet-Acid Fuchsin was then applied to stain the urethral tissue sections for $3 \mathrm{~min}$, followed by rinsing, and immersion of the tissue in phosphomolybdic acid for $45 \mathrm{~min}$. Next, the urethral tissue sections were immersed in Aniline Blue for $3 \mathrm{~min}$, followed by rinsing with distilled water for $2 \mathrm{~min}$, staining in 1\% acetic acid for $2 \mathrm{~min}$, and rinsing twice with distilled water for $2 \mathrm{~min}$. Subsequently, the urethral tissue sections were immersed in increasing ethanol concentrations for dehydration and air dried.

\section{TUNEL Assay}

To detect the number of apoptotic cell deaths in the urethral sphincter, we measured DNA fragmentation using a TUNEL apoptosis assay kit, according to the manufacturer's instructions. Briefly, urethral sphincter sections of each rat were placed in $1 \%$ Triton X-100, followed by enzymatic digestion with Proteinase K for 30 min. Then, the sections were incubated with TdT solution for $1 \mathrm{~h}$, followed by reaction with StreptavidinTRITC solution for $30 \mathrm{~min}$ in a dark, humidified chamber. The sections were incubated with DAPI for $10 \mathrm{~min}$ to stain the cell nuclei in the final step. The morphology and percentage of apoptotic cells was measured and observed via microscopy. 


\section{Oxidative Stress Marker Determination}

The concentrations of oxidative stress markers MDA, GSH-Px, total SOD, and CAT in the urethral sphincter tissue were measured in accordance with the manufacturer's instructions. The MDA concentration was detected at $532 \mathrm{~nm}$ since MDA can react with thiobarbituric acid (TBA) to reach maximum absorbance. The activity of SOD was measured at $550 \mathrm{~nm}$ based on its ability to react with xanthine and xanthine oxidase. Intracellular GSH was monitored at $412 \mathrm{~nm}$, via enzyme-catalyzed reaction product (reduced glutathione). CAT activity was detected at $405 \mathrm{~nm}$ due to the reaction of hydrogen peroxide with ammonium molybdate.

\section{Protein Extraction and Western Blotting}

Briefly, we extracted protein from frozen urethral sphincter tissue via trypan-blue in RIPA buffer containing protease inhibitors. Urethral sphincter lysates were centrifuged at $10000 \mathrm{~g} / \mathrm{min}$ for $10 \mathrm{~min}$ at $4^{\circ} \mathrm{C}$; subsequently, the supernatant was removed and used as the total protein extract. We extracted the nuclear protein with a nuclear protein extraction kit according to the manufacturer's instructions. Briefly, the urethral sphincter was cut into pieces and homogenized using a cytoplasm protein extraction reagent containing protease inhibitors. The lysates were centrifuged for $5 \mathrm{~min}$ at $10000 \mathrm{~g} / \mathrm{min}$ at $4^{\circ} \mathrm{C}$. The sediment was collected and mixed with nucleoprotein extraction reagent on ice for $30 \mathrm{~min}$, the lysates were centrifuged for $10 \mathrm{~min}$ at $10000 \mathrm{~g} / \mathrm{min}$ at $4^{\circ} \mathrm{C}$, and the supernatant was gathered and used as a nuclear protein extract. BCA protein assay was used to measure protein concentrations. The samples were run on $10 \%$ SDS-polyacrylamide gels (20 $\mu \mathrm{g} / \mathrm{lane})$, and electroblotting (200 mA) was used to transfer proteins to PVDF membranes. PVDF membranes were incubated in 5\% BSA at room temperature for $2 \mathrm{~h}$ and washed three times in TBST via 5-min washes, followed by incubation with anti-Nrf2 (1:1000), HO-1 (1: 1000), NQ01 (1: 1000), bcl-2 (1:2000), Bax $(1: 2000)$, and GAPDH $(1: 2000)$ antibodies overnight at $4^{\circ} \mathrm{C}$.

GAPDH was regarded as the internal normalizer. Subsequently, the membranes were washed three times in TBST for $10 \mathrm{~min}$ each, followed by incubation for $2 \mathrm{~h}$ with a goat anti-rabbit horseradish peroxidaseconjugated antibody $(1: 2000)$ and visualized via chemiluminescence.

\section{Statistical Analysis}

All data were displayed as the means \pm SD. SPSS 16.0 was used for data processing. Differences between groups were investigated using one-way ANOVA, and a $\mathrm{P}<0.05$ was considered statistically significant.

\section{Results}

\section{Effect of SFN on Body Weight}

The body weights of control group rats were significantly higher than the weights of the rats in both SUI and SUI+SFN groups (Table 1). The rats in the SUI+SFN group gained more weight than those in the SUI group. No other side effects were observed.

\section{Improvement of Cystometric Outcomes in SUI Rats}

The parameters of cystometry were recorded according to the urodynamic curve (Fig. 1). The bladder pressure was notably decreased in rats of the SUI group relative to those of the control group. However, the peak voiding pressure in SUI rats treated via SFN was higher than the pressure in the SUI group rats at the 4-week time point. Moreover, we observed a shorter micturition interval in SUI rats than in control rats. The interval of

Table 1. The results of body weight and cystometric parameters of rats. *Significantly different versus Control group $(\mathrm{P}<0.05)$. "Significantly different versus SUI group $(\mathrm{P}<0.05)$

\begin{tabular}{|c|c|c|c|}
\hline & Control & SUI & SUI+SFN \\
\hline \multicolumn{4}{|l|}{ Body weight } \\
\hline Preop & $173.33 \pm 16.63$ & $175.17 \pm 10.44$ & $174.67 \pm 11.22$ \\
\hline 4-week SUI & $329.83 \pm 19.41$ & $285.33 \pm 18.76^{*}$ & $297.83 \pm 12.83^{*}$ \\
\hline Peak voiding pressure $\left(\mathrm{cm} \mathrm{H}_{2} 0\right)$ & $21.08 \pm 4.40$ & $12.75 \pm 2.18^{*}$ & $18.03 \pm 5.63^{\#}$ \\
\hline Micturition interval(sec) & $86.89 \pm 10.78$ & $62.78 \pm 7.43^{*}$ & $85.11 \pm 20.70^{\#}$ \\
\hline
\end{tabular}


micturition in SUI+SFN rats was remarkably elevated compared to rats in the control group (Table 1).

Effect of SFN on SUI Induced Histological Changes in the Urethral Sphincter

HE staining revealed notably histological changes with structural damage of the urethral sphincter muscle, muscle bundle split, and thinner muscle layer in rats of the SUI group. In the other two groups, SFN exerted a protective function on the urethral sphincter. Rats in the SUI+SFN group had a urethral sphincter muscle histological image similar to those in the control group. Rats treated with SFN had a thicker muscle layer and comparatively normal histological images, as revealed via HE staining.

Masson staining showed that after SFN treatment, the proportion of muscle areas in the urethra was significantly increased in the SUI+SFN group compared to that in the SUI group. Furthermore, the collagen content in the SUI group was higher than that in the SUI+SFN group (Fig. 2).

\section{Effect of SFN on Attenuating} Oxidative Stress in SUI Rats

The level of oxidative stress was evaluated by measuring the levels of MDA, total SOD, GSH-Px, and CAT. The content of MDA in the urethral sphincter was notably increased in SUI rats compared to that in control rats $(3.31 \pm 0.74$ versus $1.50 \pm 0.34)$. The MDA level in the serum of rats of the SUI group was also significantly increased. The level of urethral MDA had decreased in SUI+SFN rats based $(2.19 \pm 0.37)$ Compared to the control group, the activities of total SOD and GSH-Px were remarkably elevated in SUI rats $(93.48 \pm 11.40$ versus $113.90 \pm 9.19$ and $149.71 \pm 13.09$ versus $189.68 \pm 18.80$, respectively). Compared to the SUI group, the activities of SOD, GSH-Px, and CAT were significantly increased in the

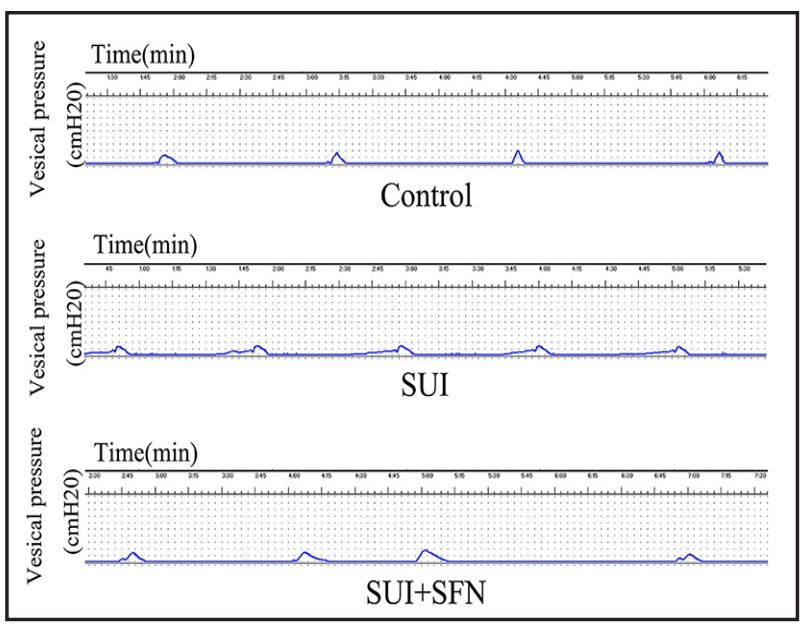

Fig. 1. The outcome of SFN therapy on urodynamic changes in rats of three groups.

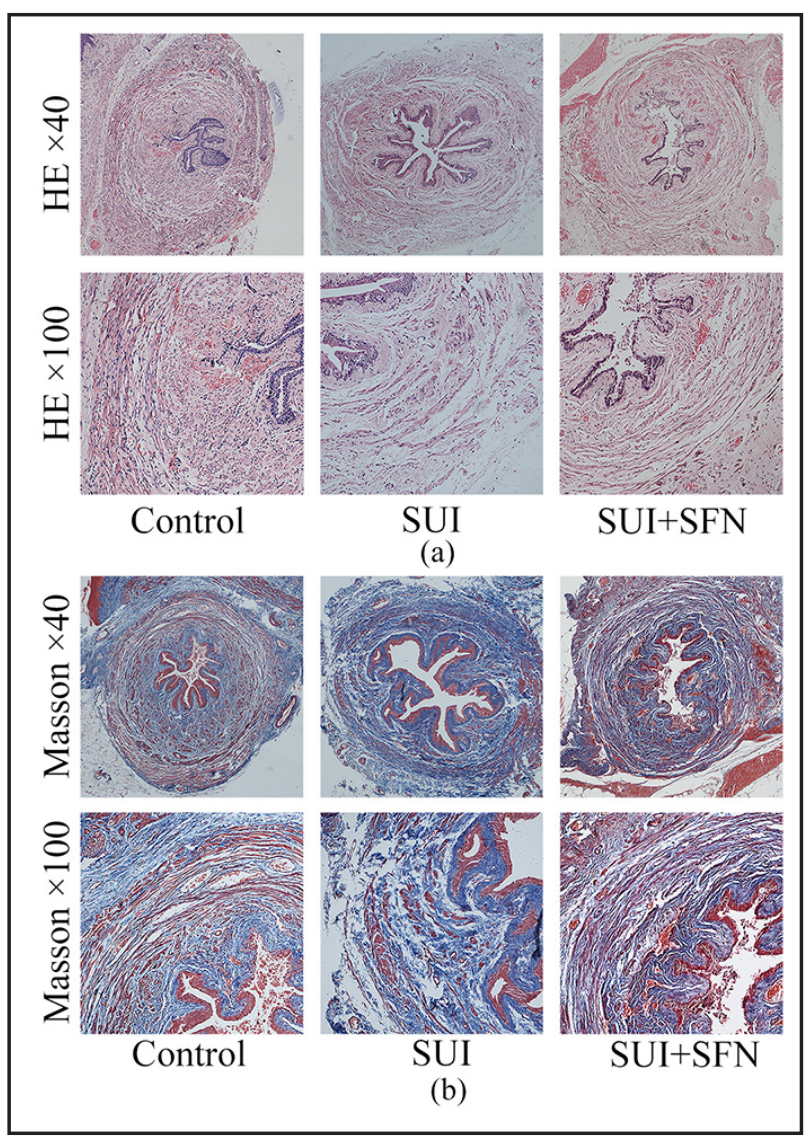

Fig. 2. (a) Effect of SFN on urethral sphincter muscle histological changes in the rats in the three groups. Original magnification $\times 40$ and $\times 100$. HE staining of urethral sphincter in control, SUI and SUI+SFN groups. (b) Effect of SFN on urethral sphincter muscle and collagen contents in rats in the three groups. Original magnification $\times 40$ and $\times 100$. Masson staining of urethral sphincter in control, SUI and SUI+SFN groups. 
Fig. 3. Effects of SFN on attenuating oxidative stress on rats in the three groups. (a) MDA level in the urethra of the rats in the three groups. (b) Serum MDA level in the urethra of the rats in the three groups. (c) The content of total SOD in the three groups. (d) The GSP-Px activity in the three groups. (e) The expression of CAT in the three groups. ${ }^{*} n=6$, $\mathrm{P}<0.05$ versus control group. ${ }^{\#} \mathrm{n}=6, \mathrm{P}<0.05$ versus SUI group.

SUI+SFN group (157.64 \pm $17.93,253.53 \pm 13.46$, and $6.88 \pm 1.15$, respectively) (Fig. 3).

Effect of SFN on
Cell Apoptosis and
Proliferation in the
Urethral Sphincter of SUI
Rats
TUNEL
revealed that SFN exerted protective effects on the cell apoptosis level of the urethral sphincter. The number of apoptotic cells in the urethral sphincter of SUI rats was markedly elevated compared to that in control

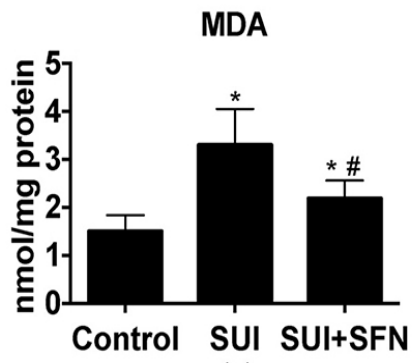

(a)

Total SOD

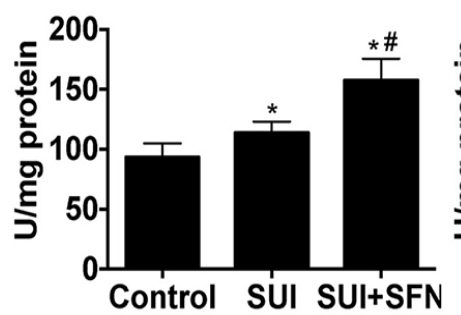

(c)

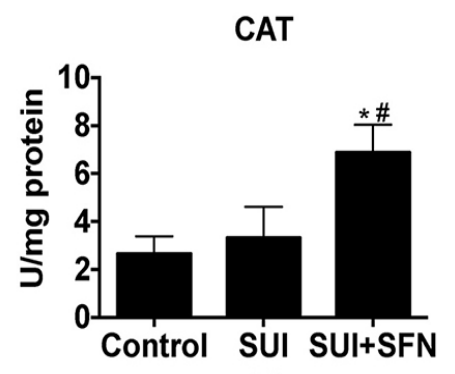

(e)

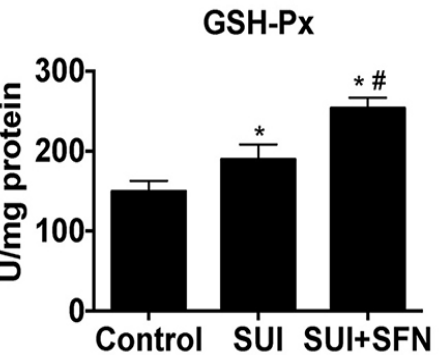

(d)

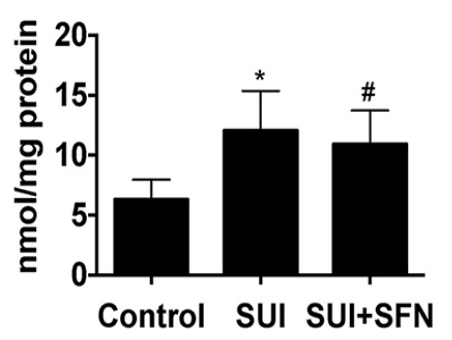

(b)

rats; however, the number of apoptotic cells in the urethral sphincter of SUI rats treated with SFN had notably decreased(3.67 $\pm 0.29 \%$ versus $12.97 \pm 1.50 \%$ and $6.73 \pm 1.00 \%$, respectively). Western blotting showed that the Bax/Bcl-2 expression ratio was significantly elevated in the urethral sphincter of SUI rats, consistent with the results of TUNEL staining. However, this ratio was decreased in SUI+SFN rats (Fig. 4).

\section{Effect of SFN on Urethral Sphincter via Activation of the Nrf2-ARE Pathway}

The Nrf2 transcription factor is a crucial mediator involved in regulating cellular antioxidative reactions [27]. We investigated the effect of SFN on the expression of Nrf2, which might be activated via SFN and its downstream target proteins. The expression level of Nrf2 was notably higher in the muscular layers of the urethral sphincters of the rats in the SUI+SFN group compared to those of the rats in the SUI group, as demonstrated via the immunohistochemical imaging. Nrf2 was mainly expressed in the Onuf's nucleus in the external sphincter. We further measured Nrf2 expression in the cell and nucleus via western blotting. The results demonstrated that the total expression of Nrf2 was significantly higher in the urethral sphincter of the SUI+SFN group compared to that in the SUI group, and total Nrf2 was significantly increased in the urethral sphincter of the rats in the SUI group compared to that in the rats in the control group. The levels of HO-1 and NOQ1 (which is the downstream target proteins of Nrf2) were evaluated to measure the antioxidative function of Nrf2. Consistent with the Nrf2 expression in the nucleus, the level of HO-1 was remarkably 
Fig. 4. Effect of SFN on cell apoptosis in rats of three groups. (a) TUNEL staining showing the cell apoptosis level in the urethral of the rats in the three groups. Original magnification $\times 100$. (b) The statistical results of the percentage of the apoptotic cells in the three groups. (c) The protein expression of Bax/Bcl2 ratio in the urethra of the rats in the three groups. (d) The statistical results of the protein expression $\mathrm{Bax} / \mathrm{Bcl} 2$ ratio in the three groups. ${ }^{*} \mathrm{P}<0.05$ versus control group. ${ }^{\#} \mathrm{P}<0.05$ versus SUI group.

higher in the SUI+SFN group compared to that in the SUI group. Moreover, the level of NQ01 in the urethral sphincter of the rats in the SUI+SFN group was noticeably higher than that in the rats in the SUI group, and the levels of NQO1 in the urethral sphincters of the rats in the SUI group were significantly elevated compared to that in the rats the

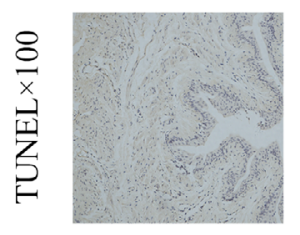

Control

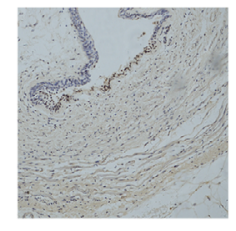

SUI

(a)

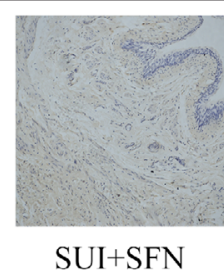

SUI+SFN

The Percentage of Apoptotic Cells

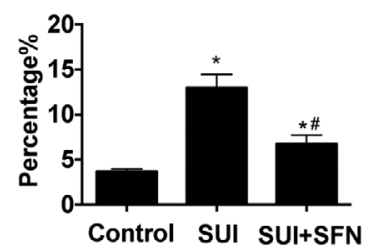

(b)

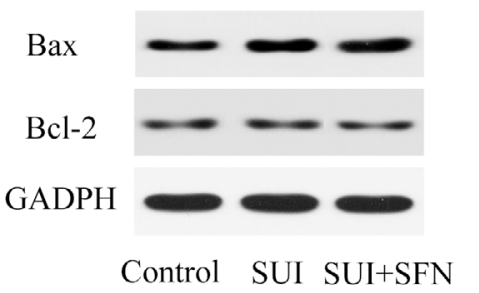

(c)

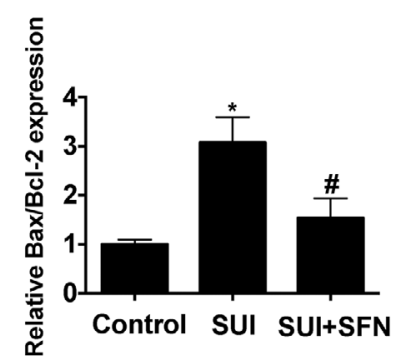

(d) control group (Fig. 5).

\section{Discussion}

While various studies have demonstrated the promising potential of SFN for the treatment of numerous diseases [28, 29], the present study may be the first to explore the feasibility of SFN for SUI treatment.

The SUI model was used in numerous studies at increasing success rates for creating abnormal voiding conditions [10,22, 23, 30-32]. This model simulated and contained the effect of delivery, birth trauma, and menopause, three factors assumed to play a central role in the pathogenesis of lower urinary tract symptomatology [23]. Prolonged elevated pressure and intravaginal distention may cause ischemia damage to the urethra sphincter. Ischemia in turn induces oxidative stress reactions, resulting in urethral relaxation and an estrogen deficiency that increases oxidative expression and decreases urethral trophicity [11]. The use of antioxidants, such as SFN, may ameliorate oxidative stress reaction-induced SUI. Herrera-Imbroda et al. [33] suggested that human SUI is a multifactorial and complex process, and Sievert et al. [10] reported that for simulating effects of difficult labor, delivery, and menopause this modified model would be the best candidate.

The goal of the present study was to evaluate the role of SFN in the treatment of SUI via the Nrf2-ARE pathway. Therefore, we detected whether SFN stimulates Nrf2 activity in the presence ofSUI, as a central regulator for cellular responses to oxidativeand electrophilicstress. To determine the amount of Nrf2 in the urethral sphincter muscle, immunohistochemical imaging and western blotting were conducted among all groups. The content of Nrf2 was significantly higher in the SUI+SFN group than in the other two groups. Total Nrf2 expressed in the SUI group was higher than that of the control group. This result demonstrated that SFN could promote the Nrf2 content during treatment in the present study (four weeks total), and the overall protection would appear to operate largely via the Nrf2 pathway. More 
importantly, the expression of Nrf2 in the nucleus was significantly increased in rats of the SUI+SFN group. In addition, rats of the SUI group expressed more Nrf2 in the nucleus than the control group. These results implied that SFN treatment and SUI induced damage could facilitate Nrf2 translocation into the nucleus to promote its targeted antioxidant genes.

Next, we used western blotting to detected transcript levels of relative genes, $\mathrm{HO}-1$, and NOQ1, which are responsible for the expression of enzymes of the antioxidant synthesis pathway in the urethral sphincter. The results showed that the levels of HO-1 and NOQ1 were higher than in the other two groups; furthermore, compared to the control group, rats of the SUI group expressed more HO-1 and NOQ1. The contents of HO-1 and NOQ1 were consistent with the Nrf2 content in the nucleus. In addition, we tested the total content of CAT, GSH-Px, and SOD and the results were identical to the expression levels of HO-1 and NOQ1. Overall, The 30-day intraperitoneal injections of SFN could sustained promote the translocation of Nrf2 into the nucleus. Rats of the SUI group receiving vaginal distention damage may trigger the self protect function by the translocation of Nrf2 into the nucleus. The Nrf2 in the nucleus region could connect with AREs to boost the cytoprotective genes, such as NQ01, HO-1, to produce SOD, GSH-Px, CAT to exert antioxidative function.

To reflect the therapeutic effect of SFN on SUI, we performed urodynamic studies in conscious rats. The method of measuring urodynamic studies was consistent with Lin et al. [34]. We evaluated

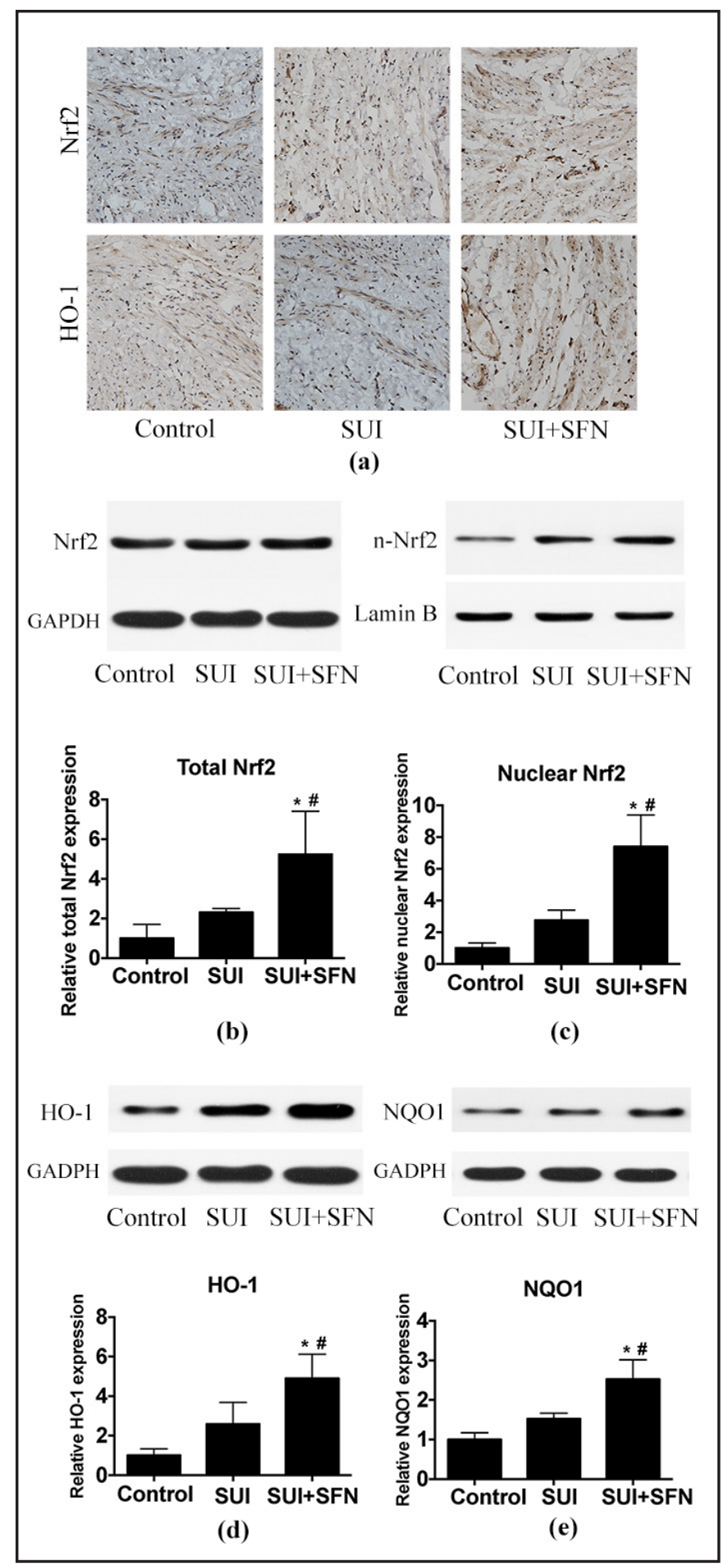

Fig. 5. Effect of SFN on the Nrf2-ARE pathway in the three groups. (a) Immunohistochemical staining of Nrf2 and HO-1 in the urethra of the rats in the three groups. Original magnification $\times 200$. (b) The total Nrf2 expression in the three groups. (c) The nuclear Nrf2 expression in the urethra of the rats in the three groups. (d) The expression of HO-1 in the three groups. (e) The expression of NQ01 in the three groups. ${ }^{*} \mathrm{P}<0.05$ versus control group. ${ }^{*} \mathrm{P}<0.05$ versus SUI group. 
cystometric parameters, such as bladder capacity, maximal pressure, micturition interval, and compliance. Bladder capacity, micturition interval, and leak point pressure showed improvement, to some extent, through treatment with SFN in BOO rats. This result indicated that SFN could protect the urinary function of rats in an effective manner. Furthermore, daily intraperitoneal injections of SFN $(0.5 \mathrm{mg} / \mathrm{kg})$ have previously been demonstrated as effective [35]. The urodynamic outcome was consistent with the histological changes in the urethral sphincter. HE staining and Masson staining revealed a normal-appearing circular muscle mass morphology at the urethral sphincter region in the SUI+SFN group. However, the SUI group revealed only high collagen content, scant muscle morphology, and wavy sphincters. Lin et al. applied adipose tissue-derived stem cells to treat postpartum vaginal balloon dilation in bilateral ovariectomy induced SUI model rats. This author reported that after stem cell treatment, normal voiding rats had remarkably increased muscle content and muscle/collagen ratio compared to the abnormal voiding rats, which is similar to the findings of the present study. Another study reported that the muscle bundles of urethral muscle of virgin female C57BL/ 6 appeared wavy and disrupted following vaginal distention [36]. Compared to the present model, although the previous model could simulate birth trauma, it lacks the simulation of delivery and menopause. A clinical study of detrusor and urethral musculature of ultrastructural imaging in women suffering from urinary incontinence revealed that urethral muscle cells almost entirely lack direct cell-to-cell communication and are separated by several microns [37]. Strasser et al. [38] necropsied 23 clinically healthy individuals and found an age-dependent occurrence of rhabdosphincter apoptosis. Continuous morphological and functional changes of the striated muscle cells of the rhabdosphincter with increasing age may account for the higher urinary incontinence incidence. Kamo et al. [39] considered the contraction of external urethral sphincter to be essential to preserve urinary continence during elevated abdominal pressure.

The western blot results for bax and bcl-2 revealed that SUI rats had more apoptotic cells. However, a previous study reported that the apoptotic cells were mainly located in the epithelium and submucosa and few apoptotic cells were found within the muscle layer of the SUI model [23], consistent with the present results. Therefore, we suggest that SFN may improve urinary function, not by restraining muscle cell apoptosis, but by preserving muscle function. Similar to other reported studies [29] [40], SFN could improve muscle contractile function, preserve the normal muscle morphology, protect muscles from oxidative damage, and decrease collagen expression. The precise mechanism through which SFN influences the urethral sphincter muscle should be further explored.

\section{Disclosure Statement}

The authors declare that they have no conflicts of interest

\section{Acknowledgements}

The work was sponsored by the National Natural Science Foundation of China (Grant No. 81402089, 81702498); Clinical Research Program of 9th People's Hospital, Shanghai Jiao Tong University School of Medicine (JYLJ005); Program for Outstanding Medical Academic Leader; Integrated Traditional Chinese and Western Medicine of Shanghai (ZHYYZXYJHZX-1-03); the Innovation Project of Shanghai Jiao Tong University School of Medicine in China (16XJ21003). 


\section{Cellular Physiology Cell Physiol Biochem 2017;44:1912-1922 \begin{tabular}{l|l|l} 
and Biochemistry 10.1159/000485880 & $\begin{array}{l}\text { (c) 2017 The Author(s). Published by S. Karger AG, Basel } \\
\text { www.karger.com/cpb }\end{array}$
\end{tabular}}

Wan et al.: Treating Stress Urinary Incontinence

\section{References}

1 Norton P, Brubaker L: Urinary incontinence in women. Lancet 2006;367:57-67.

2 Retzky SS, Rogers RM Jr: Urinary incontinence in women. Clin Symp 1995;47:2-32.

-3 Brown JS, Grady D, Ouslander JG, Herzog AR, Varner RE, Posner SF: Prevalence of urinary incontinence and associated risk factors in postmenopausal women. Heart \& Estrogen/Progestin Replacement Study (HERS) Research Group. Obstet Gynecol 1999;94:66-70.

4 Thom D: Variation in estimates of urinary incontinence prevalence in the community: effects of differences in definition, population characteristics, and study type. J Am Geriatr Soc 1998;46:473-480.

5 Shamliyan TA, Kane RL, Wyman J, Wilt TJ: Systematic review: randomized, controlled trials of nonsurgical treatments for urinary incontinence in women. Ann Intern Med 2008;148:459-473.

6 Kerr LA: Bulking agents in the treatment of stress urinary incontinence: history, outcomes, patient populations, and reimbursement profile. Rev Urol 2005;7:S3-s11.

-7 Sweat SD, Lightner DJ: Complications of sterile abscess formation and pulmonary embolism following periurethral bulking agents. J Urol 1999;161:93-96.

8 Gilchrist AS, Rovner ES: Managing complications of slings. Curr Opin Urol 2011;21:291-296.

9 Kim BS, Chun SY, Lee JK, Lim HJ, Bae JS, Chung HY, Atala A, Soker S, Yoo JJ, Kwon TG: Human amniotic fluid stem cell injection therapy for urethral sphincter regeneration in an animal model. BMC Med 2012;10:94.

10 Sievert KD, Emre Bakircioglu M, Tsai T, Dahms SE, Nunes L, Lue TF: The effect of simulated birth trauma and/or ovariectomy on rodent continence mechanism. Part I: functional and structural change. J Urol 2001;166:311-317.

11 Chen HY, Chen WC, Lin YN, Chen YH: Synergistic effect of vaginal trauma and ovariectomy in a murine model of stress urinary incontinence: upregulation of urethral nitric oxide synthases and estrogen receptors. Mediators Inflamm 2014;2014:314846.

12 Canda AE, Cinar MG, Turna B, Sahin MO: Pharmacologic targets on the female urethra. Urol Int 2008;80:341-354.

13 Chen HY, Lin YN, Chen YH, Chen WC: Stress urinary incontinence following vaginal trauma involves remodeling of urethral connective tissue in female mice. Eur J Obstet Gynecol Reprod Biol 2012;163:224229.

14 Chen YH, Lin YN, Chen WC, Hsieh WT, Chen HY: Treatment of stress urinary incontinence by cinnamaldehyde, the major constituent of the chinese medicinal herb ramulus cinnamomi. Evid Based Complement Alternat Med 2014;2014:280204.

15 Kaspar JW, Niture SK, Jaiswal AK: Nrf2:INrf2 (Keap1) signaling in oxidative stress. Free Radic Biol Med 2009;47:1304-1309.

16 Keum YS, Choi BY: Molecular and chemical regulation of the Keap1-Nrf2 signaling pathway. Molecules 2014;19:10074-10089.

17 Singh P, Sharma R, McElhanon K, Allen CD, Megyesi JK, Benes H, Singh SP: Sulforaphane protects the heart from doxorubicin-induced toxicity. Free Radic Biol Med 2015;86:90-101.

18 Fahey JW, Zhang Y, Talalay P: Broccoli sprouts: an exceptionally rich source of inducers of enzymes that protect against chemical carcinogens. Proc Natl Acad Sci U S A 1997;94:10367-10372.

-19 Talalay P, Fahey JW, Holtzclaw WD, Prestera T, Zhang Y: Chemoprotection against cancer by phase 2 enzyme induction. Toxicol Lett 1995;82-83:173-179.

20 Bai Y, Cui W, Xin Y, Miao X, Barati MT, Zhang C, Chen Q, Tan Y, Cui T, Zheng Y, Cai L: Prevention by sulforaphane of diabetic cardiomyopathy is associated with up-regulation of Nrf2 expression and transcription activation. J Mol Cell Cardiol 2013;57:82-95.

21 Piao CS, Gao S, Lee GH, Kim DS, Park BH, Chae SW, Chae HJ, Kim SH: Sulforaphane protects ischemic injury of hearts through antioxidant pathway and mitochondrial K(ATP) channels. Pharmacol Res 2010;61:342348.

22 Hayashi N, Bella AJ, Wang G, Lin G, Deng DY, Nunes L, Lue TF: Effect of extended-term estrogen on voiding in a postpartum ovariectomized rat model. Can Urol Assoc J 2007;1:256-263.

23 Resplande J, Gholami SS, Graziottin TM, Rogers R, Lin CS, Leng W, Lue TF: Long-term effect of ovariectomy and simulated birth trauma on the lower urinary tract of female rats. J Urol 2002;168:323-330. 


\section{Cellular Physiology Cell Physiol Biochem 2017;44:1912-1922

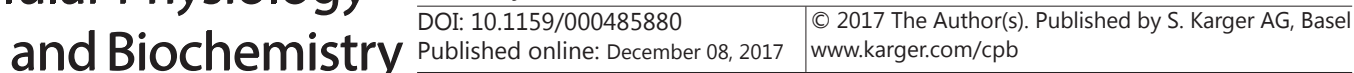 \\ Wan et al.: Treating Stress Urinary Incontinence}

24 Miyazaki N, Yamaguchi O, Nomiya M, Aikawa K, Kimura J: Preventive Effect of Hydrogen Water on the Development of Detrusor Overactivity in a Rat Model of Bladder Outlet Obstruction. J Urol 2016;195:780787.

25 Tu DD, Seth A, Gil ES, Kaplan DL, Mauney JR, Estrada CR Jr: Evaluation of biomaterials for bladder augmentation using cystometric analyses in various rodent models. J Vis Exp 2012;(66).pii3981.

-26 Liu C, Xu H, Fu S, Chen Y, Chen Q, Cai Z, Zhou J, Wang Z: Sulforaphane Ameliorates Bladder Dysfunction through Activation of the Nrf2-ARE Pathway in a Rat Model of Partial Bladder Outlet Obstruction. Oxid Med Cell Longev 2016;2016:7598294.

27 Scannevin RH, Chollate S, Jung MY, Shackett M, Patel H, Bista P, Zeng W, Ryan S, Yamamoto M, Lukashev M, Rhodes KJ: Fumarates promote cytoprotection of central nervous system cells against oxidative stress via the nuclear factor (erythroid-derived 2)-like 2 pathway. J Pharmacol Exp Ther 2012;341:274-284.

28 Bhakkiyalakshmi E, Sireesh D, Rajaguru P, Paulmurugan R, Ramkumar KM: The emerging role of redoxsensitive Nrf2-Keap1 pathway in diabetes. Pharmacol Res 2015;91:104-114.

29 Sun C, Yang C, Xue R, Li S, Zhang T, Pan L, Ma X, Wang L, Li D: Sulforaphane alleviates muscular dystrophy in mdx mice by activation of Nrf2 J Appl Physiol (1985) 2015;118:224-237.

30 Banie L, Lin G, Ning H, Wang G, Lue TF, Lin CS: Effects of estrogen, raloxifene and levormeloxifene on alpha1A-adrenergic receptor expression. J Urol 2008;180:2241-2246.

31 Lin G, Shindel AW, Banie L, Deng D, Wang G, Hayashi N, Lin CS, Lue TF: Molecular mechanisms related to parturition-induced stress urinary incontinence. Eur Urol 2009;55:1213-1222.

-32 Tantiwongse K, Fandel TM, Wang G, Breyer BN, Walsh TJ, Bella AJ, Lue TF: The potential of hormones and selective oestrogen receptor modulators in preventing voiding dysfunction in rats. BJU Int 2008;102:242246.

-33 Herrera-Imbroda B, Lara MF, Izeta A, Sievert KD, Hart ML: Stress urinary incontinence animal models as a tool to study cell-based regenerative therapies targeting the urethral sphincter. Adv Drug Deliv Rev 2015;82-83:106-116.

-34 Lin G, Wang G, Banie L, Ning H, Shindel AW, Fandel TM, Lue TF, Lin CS: Treatment of stress urinary incontinence with adipose tissue-derived stem cells. Cytotherapy 2010;12:88-95.

-35 Wang Y, Zhang Z, Guo W, Sun W, Miao X, Wu H, Cong X, Wintergerst KA, Kong X, Cai L: Sulforaphane reduction of testicular apoptotic cell death in diabetic mice is associated with the upregulation of Nrf2 expression and function. Am J Physiol Endocrinol Metab 2014;307:E14-23.

-36 Lin YH, Liu G, Li M, Xiao N, Daneshgari F: Recovery of continence function following simulated birth trauma involves repair of muscle and nerves in the urethra in the female mouse. Eur Urol 2010;57:506-512.

37 Hale DS, Benson JT, Brubaker L, Heidkamp MC, Russell B: Histologic analysis of needle biopsy of urethral sphincter from women with normal and stress incontinence with comparison of electromyographic findings. Am J Obstet Gynecol 1999;180:342-348.

38 Strasser H, Tiefenthaler M, Steinlechner M, Bartsch G, Konwalinka G: Urinary incontinence in the elderly and age-dependent apoptosis of rhabdosphincter cells. Lancet 1999;354:918-919.

-39 Kamo I, Torimoto K, Chancellor MB, de Groat WC, Yoshimura N: Urethral closure mechanisms under sneeze-induced stress condition in rats: a new animal model for evaluation of stress urinary incontinence. Am J Physiol Regul Integr Comp Physiol 2003;285:R356-365.

-40 Fernandes RO, De Castro AL, Bonetto JH, Ortiz VD, Muller DD, Campos-Carraro C, Barbosa S, Neves LT, Xavier LL, Schenkel PC, Singal P, Khaper N, da Rosa Araujo AS, Bello-Klein A: Sulforaphane effects on postinfarction cardiac remodeling in rats: modulation of redox-sensitive prosurvival and proapoptotic proteins. J Nutr Biochem 2016;34:106-117. 